DOI: https://doi.org/10.24297/jab.v12i0.8253

\title{
The Effect of ZJXG Decoction on The Serum Level of Growth Hormone and The Expression of GH Receptor in Callus
}

\author{
Zhen Zhou'1, Guanxi Wang ${ }^{2}$,Zhaogang Liu',Yunliang Guo ${ }^{3}$ \\ School of Basic Medicine, Qingdao University, Qingdao 266071, China \\ Department of Imageology, Songshan Hospital, Qingdao University, Qingdao 266021, China \\ Institute of Integrative Medicine, Qingdao University, Qingdao 266021,China \\ Inlyfly@163.com; Lzg532@163.com, wangguanxi@163.com,guoqdsd@163.com
}

\begin{abstract}
The aim of experiment was to investigate the effects of Zhuangjin XuGu decoction (ZJXG decoction) on growth hormone $(\mathrm{GH})$ serum and $\mathrm{GH}$ receptor (GHR) expression in callus. The femur fracture animal models were generated in 72 Wistar rats by cutting femur transversely at the middle point. The rats models were administered orally ZJXG decoction for 28 days. Anatomy, X-ray and hematoxylin- eosin (HE) staining were used to observe the healing process in rats. The expression of growth hormone receptor (GHR) in fibroblasts and osteoblasts in callus was evaluated by immunohistochemical assay (IHC). The serum level of $\mathrm{GH}$ was passed by enzyme linked immunosorbent assay (ELISA). Anatomy, $\mathrm{X}$-ray and $\mathrm{H}$-E staining indicated that the fibrous callus at the fractureend increased and the fibrous granular tissue changed gradually to fibrous, cartilaginous and osseous callus. IHC and ELISA showed that after 28 days of ZJXG Decoction treatment, that GH in the fibroblasts and osteoblasts of callus and their serum level increased significantly. These results suggested that ZJXG decoction could enhance the fracture healing by enhancing GH activity and promoting the expression of GHR in the fibroblasts and osteoblasts of callus in rats.
\end{abstract}

Key words: ZJXG Decoction; Femur Fracture; Growth Hormone (GH); Growth Hormone Receptor (GHR), Rats

\section{Introduction}

Growth hormone $(\mathrm{GH})$ is a polypeptide secreted by pituitary gland and a major regulator of collagen growth[1]. After fracture, pituitary eosinophils were in a high functional state, GH expression increased, which affected the differentiation of osteoblasts, stimulated alkaline phosphatase (ALP) activity, ALP activity could reflect the function of osteoblasts [2], thus promoting osteocalcin synthesis and fracture healing [3]. Growth hormone receptor (GHR) is widely distributed in various tissues of organisms (including callus). From embryonic development to prenatal stage, The gene expression levels of GHR in all tissues was low. After birth, the level was significantly increased in a short time, especially in liver, kidney, heart and muscle..In addition, the expression level also increased during pregnancy[4]. However, it has not been reported whether the GHR expression on the surface of osteoblast membrane in callus tissue after fracture is enhanced. Previous studies of our group have shown that ZJXG Decoction can promote fracture healing [5], but its mechanism is not very clear [6]. This experiment attempts to observe the effect of traditional Chinese medicine ZJXG Decoction on serum GH level and GHR expression in callus tissue after femoral fracture in rats, and to explore its role and possible mechanism in promoting fracture healing in rats. 


\section{Materials and Methods}

1.1 Animal Grouping: Total of 72 male adult Wistar rats purchased from Experiment Animal Center of Qingdao Drug Inspection Institute, SCXK (LU) 20120010), weighted 230-250g. All animals were fed adaptively for 7 days and then divided randomly into Sham-operated group (24 rats), control group (24 rats) and treatment group (24 rats).

1.2.1 Establishment of Experimental Animal Models: The rats were anaesthetized by intraperitoneal injection of $10 \%$ Chloral hydrate $(300 \mathrm{mg} / \mathrm{kg}$ ), and then restrained in a prone position for operation. The femoral fracture model was established by cutting the femur transversely with low speed dental drill (JBX-NE22, NSK Co. Ltd. Japan at the middle section (about $0.5 \mathrm{~cm}$ below the greater trochanter), fixed with intramedullary Kirschner wires (diameter $1.0 \mathrm{~mm}$, Shanghai Medical Apparatus Co. Ltd.). The Sham-operated group was subjected to the same procedure except without cutting femur. Animals were allowed to drink and eat freely after surgery. The survival rate was $100 \%$.

1.2.2 Intervention Treatment: According to Shang Ke Da Cheng written by Zhao Lian of the Qing Dynasty in ancient China and the requirements of document No. 3 of Chinese Traditional Medicine (2009), ZJXG Decoction was prepared in our hospital preparation room. The final solution was $250 \mathrm{ml}$, containing $125 \mathrm{~g}$ of crude medicine, and the concentration was $0.5 \mathrm{~g} / \mathrm{ml}$. Stored at $-20^{\circ} \mathrm{C}$. According to our previous research [6], ZJXG Decoction was given in daily oral doses at $1.25 \mathrm{~g} / \mathrm{kg}(2.5 \mathrm{ml} / \mathrm{kg})$ for 28 consecutive days. The Sham-operated group and the control group were given the same amount of physiological saline at the same times.

1.3 Evaluation Indexes Six rats were selected from each group to be observed in 7, 14, 21, 28 days after treatment.

1.3.1 X-ray: X-ray films (GE Revolution RE/d type, USA) of fracture femur were used to observe the processes of fracture healing.

1.3.2 Anatomical Observation: The anesthetized rats were then sacrificed and the femurs were taken out, washed of the remaining blood with normal saline for gross observation.

1.3.3 H-E Staining: Brought out the femur, removed the excess soft tissue, washed with saline, fixed it with $4 \%$ polyformaldehyde for 24 hours, soaked it in distilled water for 4 hours, and then decalcified it with 20\% EDTA solution for 15 days. Then the callus tissue was cut off, dehydrated with ethanol, transparentized by xylene, embedded in paraffin, and sliced continuously along the longitudinal axis of the femur, The thickness was $5 \mu \mathrm{m}$. The callus was bonded to glass slides, which were stained by Hematoxylin and eosin (HE) for the observation of the histological structure of callus.

1.3.4 Enzyme-Linked Immunosorbent Assay (ELISA): After treatment, rats were fasted for 12 hours, $4 \mathrm{ml}$ blood samples were obtained from abdominal aorta, and centrifuged for $10 \mathrm{~min}$ at $4000 \mathrm{rpm}$. Serum $\mathrm{GH}$ concentration was determined by double-antibody sandwich ELISA, expressed in $\mathrm{ng} / \mathrm{ml}$.

1.3.5 Immunohistochemical Staining: The positive cells were observed under 400-fold light microscopy. The negative control sections were stained with $0.01 \mathrm{~mol} / \mathrm{L}$ PBS instead of primary antibody, and no positive staining was found.. Five non-overlapping visual fields were randomly selected for observation under 400-fold optical microscope. Image-Pro Plus image processing and analysis system was used to analyze the absorbance value 
(A) of gray scale analysis. Absorption value (A) was measured by Image-Pro Plus image processing and analysis system. The expression level was expressed by subtracting background $A$ from positive cell $A$, and the mean value was taken.

1.3.6 Tartrate-Resistant Acid Phosphatase (TRAP) Staining : Bone marrow stromal cells were isolated by routine method. After 7 days of culture, the cells were cultured on slides and fixed in $25 \mathrm{~g} / \mathrm{L}$ glutaraldehyde for 7 minutes at $4^{\circ} \mathrm{C}$ and rinsed with distilled water for 3 times. Mix $0.125 \mathrm{ml} \mathrm{GBC}$ solution and $0.125 \mathrm{ml}$ nitrite solution gently for 30 seconds and rest for 2 minutes. The mixed solution and $0.125 \mathrm{ml}$ naphthol AS-BI phosphate solution, $0.5 \mathrm{ml}$ acetic acid solution, $0.25 \mathrm{ml}$ tartaric acid solution were added into the preheated $11.25 \mathrm{ml}$ tri-distilled water respectively to prepare the dyeing solution. The slides were incubated in the dye solution at $37^{\circ} \mathrm{C}$ for 1 hour and rinsed with tri-distilled water for three times. Under optical microscope, TRAP staining was positive for wine-red multinucleated giant cells ( $>3$ nuclei), which were osteoclasts. The relationship between osteoclasts and GHR distribution was observed and analyzed.

1.4 Statistical Processing: All results were expressed as $\bar{X} \pm S$ and analyzed using SPSS Version 17.0.

\section{Results}

2. 1 X-Ray Films: Sham-operated rats' femoral bone cortex were complete and continuous, with clear bone marrow cavities (Figure 1a). The model group rats' fracture line was clear on the first day, with the surrounding soft tissue swelled (Figure 1f). Seven days after bone fracture, fracture line of the rats in control group became more clear (Figure 1b); on the fourteenth day, the fracture line was slightly fuzzy (Figure 1c); on the twenty-first day, calcium salt deposition began (Figure 1d); on twenty-eighth day, bone callus formatted(Figure 1e). In 7-21 days after fracture (Figure 1g-i), callus structure for the treatment group's rats showed no significant difference from that for the control group's rats at each time point, but on the twenty-eighth day, treatment group (Figure 1j) callus structure turned better than the control group's (Figure 1e), with the images becoming clearer.

\section{Figure 1 The X-Ray Films of Femur Fracture Rats}

a. Sham-operated G.; b. Control G. 7d; c. Control G. 14d; d. Control G. 21d; e. Control G. 28d;

f. Model G. 1d; g. Treatment G. 7d; h. Treatment G. 14d; I Treatment G. 21d; j. Treatment G. 28d.

\subsection{Gross Anatomy}

Cortical bone was complete in Sham-operated group rats (Figure 2a), while the fracture fragments were separately distributed in model group rats (Figure 2f). The broken ends were surrounded by a small amount of soft fibrous granulation tissue in 7 days after fracture (Figure 2b); in 14 days, they were packaged by a large number of pliable fibrous calluses in a spindle shape (Figure 2c). In 21 days, a large number of fibrous calluses was packaged and hardened (Figure $2 \mathrm{~d}$ ). Fibrous callus was then gradually replaced by cartilage and bone callus in 28 days (Figure 2e). There was no statistical difference between treatment group and control group during 721 days at the corresponding time point (Figure $2 \mathrm{~g}-\mathrm{i}$ ), but callus calcification in treatment group (Figure $2 \mathrm{j}$ ) was significantly better than that in control group (Figure 2e). 

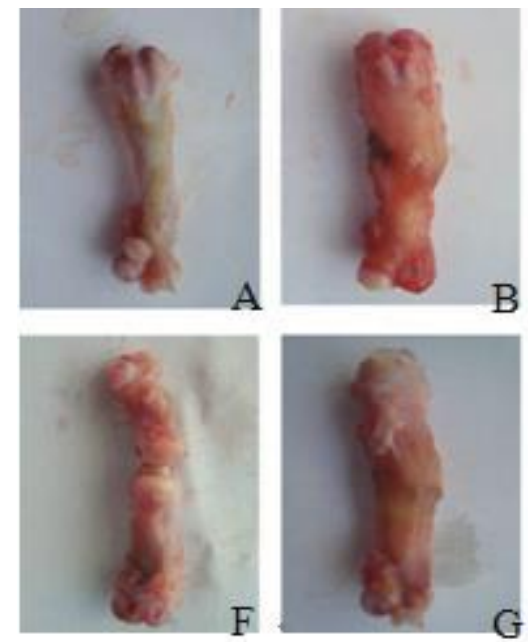

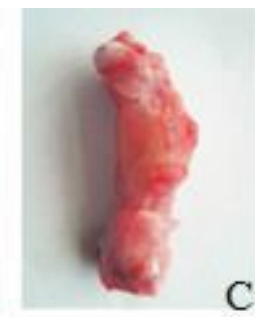

$\mathrm{C}$

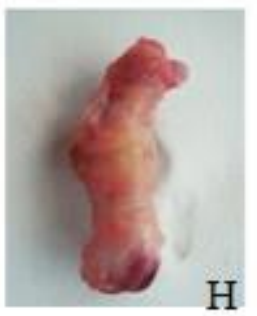

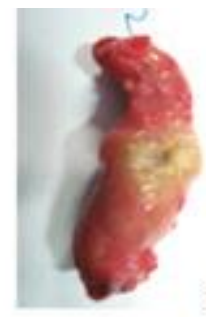

$\mathrm{D}$
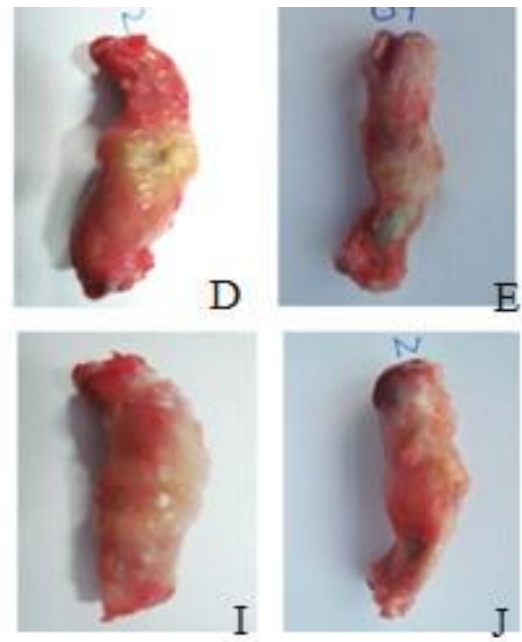

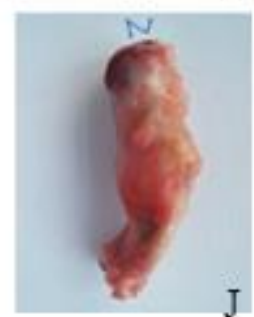

Figure 2 The Gross Anatomy of Femur in Fracture Rats

A. Sham-operated G.; b. Control G. 7d; c. Control G. 14d; d. Control G. 21d; e. Control G. 28;

f. Model G.1d; g. Treatment G. 7d; h. Treatment G. 14d; i. Treatment G. 21d; j. Treatment G. 28d.

2.3 H-E Staining: The bone structure of the sham-operated group was normal (Figure 3A). On the day of modeling, the fracture ends were filled with hematoma tissue (Figure 3F). In the control group, inflammatory cells infiltrated at the fracture ends 7 days after fracture (Figure 3B). Fibroblasts proliferated from sub periosteum into the fracture space, fibroblast granulation tissue was mixed with a small amount of osteoblasts. There was no significant difference between the treatment group and the control group at day 14 (Figure 3C). There was no significant difference between the treatment group and the control group in the growth of broken end fibrous tissue, the number of osteoblasts increased, fibrous callus formation and a small amount of cartilage callus. On the 21st day (Figure 3D), osteoclasts and osteoblasts were active, and bone remodeling lacunae and scattered trabecular formation were observed. On the 28th day (Figure 3E), trabecular formation was clearly visible and regularly arranged, and bone cortex and bone marrow cavity were visible. There was no significant difference in the skeleton structure between the treatment group and the model group at 7-21 days (Figure 3G, $\mathrm{H}, \mathrm{I})$, but the arrangement of osteocytes in the treatment group was relatively neat and clear (Figure $3 \mathrm{~J}$ ), which was significantly better than that in the control group (Figure 3E). 

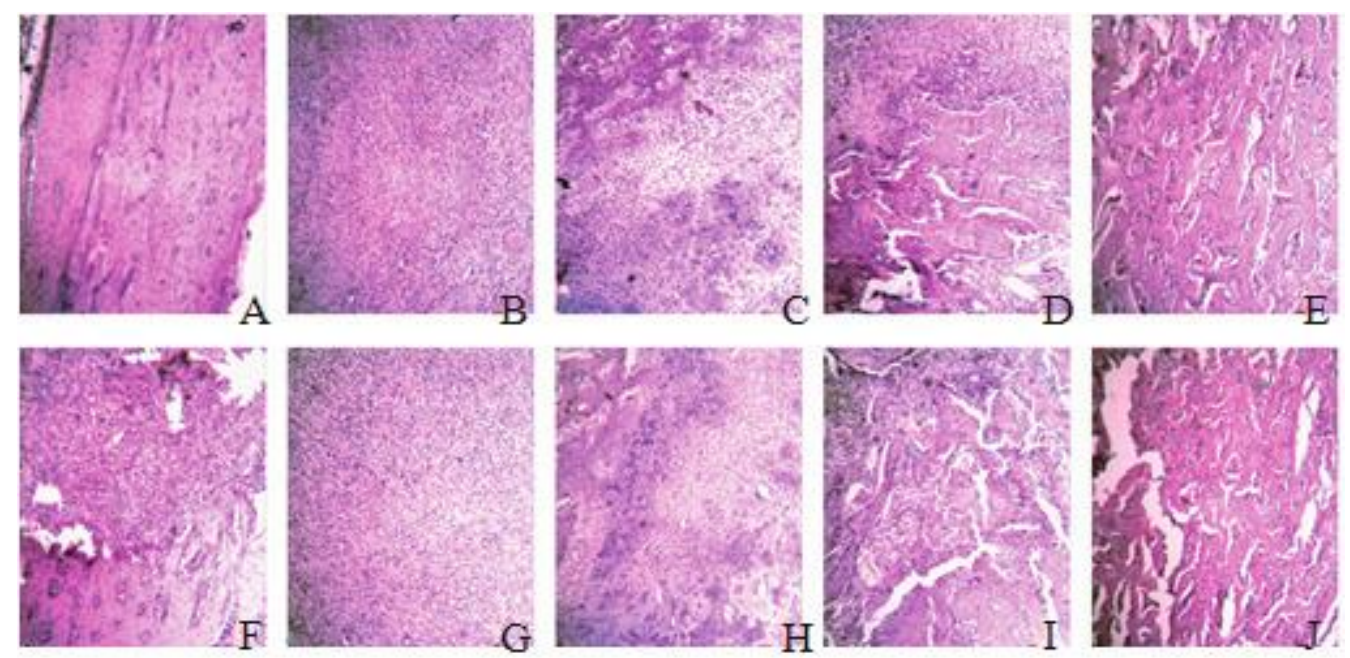

Figure 3. Pathological Observation of Callus at Fracture Site in Rats (H-E Staining *200)

A. Sham group; B. control G.7d; C. control G.14d; D. control G.21d; E. control G.28d;

F. Modeling G.1d; G. treatment G.7d; H. treatment G.14d; I. treatment G.21d; J. treatment G.28d

\subsection{Serum GH Level}

ELISA detection showed that there was no significant change in serum GH level in Sham-operated group at 7, 14,21 and 28 days after operation, $P>0.05$. There was no significant change in serum $\mathrm{GH}$ level in control group and treatment group at 7, 14 and 21 days after operation, $\mathrm{P}>0.05$, but it was significantly higher than that in Sham-operated group, $\mathrm{P}<0.05$, and decreased significantly at 28 days, $\mathrm{P}<0.05$. There was no significant difference in serum $\mathrm{GH}$ level between control group and treatment group on 7, 14 and 21 days after operation $(P>0.05)$, but the serum $\mathrm{GH}$ level in treatment group was significantly higher than that in sham-operated group and control group on 28 days after operation $(P<0.05)$. See Table 1 and 2.

Table 1. Serum GH Content (Mean Standard Deviation)

\begin{tabular}{lccccc}
\hline Groups & $\mathrm{n}$ & $7 \mathrm{~d}$ & $14 \mathrm{~d}$ & $21 \mathrm{~d}$ & $28 \mathrm{~d}$ \\
\hline Sham group & 6 & $14.05 \pm 0.61$ & $14.08 \pm 0.67$ & $14.05 \pm 0.61$ & $14.36 \pm 0.63$ \\
& & & & & \\
Control group & 6 & $17.63 \pm 0.75^{\mathrm{a}}$ & $17.37 \pm 0.64^{\mathrm{a}}$ & $17.75 \pm 0.70^{\mathrm{a}}$ & $14.53 \pm 0.56^{\mathrm{c}}$ \\
& & & & & \\
Treated group & 6 & $16.82 \pm 0.73^{\mathrm{a}}$ & $16.79 \pm 0.55^{\mathrm{a}}$ & $17.38 \pm 0.64^{\mathrm{a}}$ & $16.54 \pm 0.65^{\mathrm{ab}}$ \\
\hline
\end{tabular}

as compared with sham-operated group, $P<0.05 ;{ }^{b}$ Compared with control group, $P<0.05$; $\quad{ }^{c}$ Compared with 21 days $<0.05$ 
Table 2. Serum GH Content (Mean +Standard Deviation)

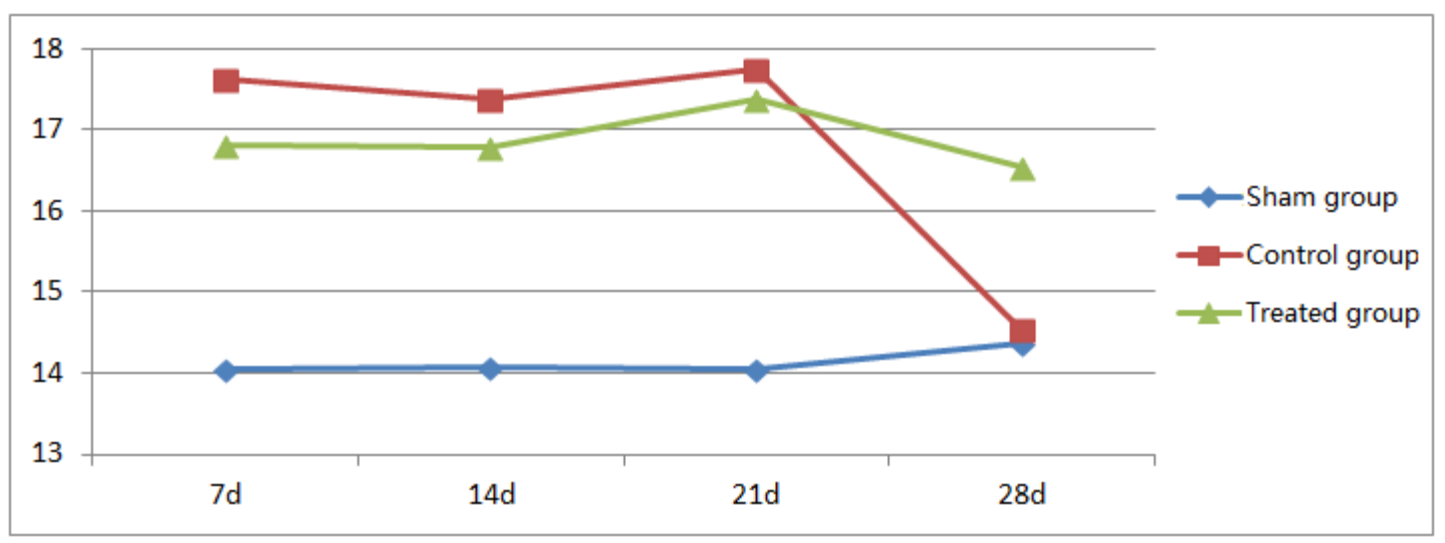

\subsection{GHR Expression Intensity in Callus Tissue}

Immunohistochemical examination showed that there was no significant change in GHR expression intensity in callus tissue of rats in sham-operated group at 7, 14, 21 and 28 days after operation, P $>0.05$. There was no significant change in callus tissue of rats in control group and treatment group at 7,14 and 21 days after operation, $P>0.05$, but it was significantly higher than that in sham-operated group, $P<0.05$, and decreased significantly at 28 days, $P<0.05$. There was no significant difference in GHR expression intensity between control group and treatment group on 7, 14 and 21 days after operation $(P>0.05)$, but GHR expression intensity in treatment group was significantly higher than that in Sham-operated group and control group on 28 days after operation $(P<0.05)$. See tables 3 and 4.

Table 3. GHR Expression Intensity in Callus Tissue (Mean \pm Standard Deviation)

\begin{tabular}{lcccll}
\hline Groups & $\mathrm{n}$ & $7 \mathrm{~d}$ & $14 \mathrm{~d}$ & $21 \mathrm{~d}$ & $28 \mathrm{~d}$ \\
\hline Sham group & 6 & $0.25 \pm 0.05$ & $0.26 \pm 0.07$ & $0.28 \pm 0.03$ & $0.27 \pm 0.03$ \\
Control group & 6 & $0.31 \pm 0.05^{\mathrm{a}}$ & $0.39 \pm 0.04^{\mathrm{a}}$ & $0.41 \pm 0.03^{\mathrm{a}}$ & $0.32 \pm 0.06^{\mathrm{c}}$ \\
Treated group & 6 & $0.32 \pm 0.03^{\mathrm{a}}$ & $0.41 \pm 0.05^{\mathrm{a}}$ & $0.40 \pm 0.04^{\mathrm{a}}$ & $0.42 \pm 0.05^{\mathrm{ab}}$ \\
\hline
\end{tabular}

as compared with sham-operated group, $P<0.05 ; \quad{ }^{b}$ Compared with control group, $P<0.05$; ${ }^{c}$ Compared with 21 days, $P<0.05$ 
Table 4. The Intensity of GHR Expression in Callus Tissue (Mean \pm Standard Deviation)

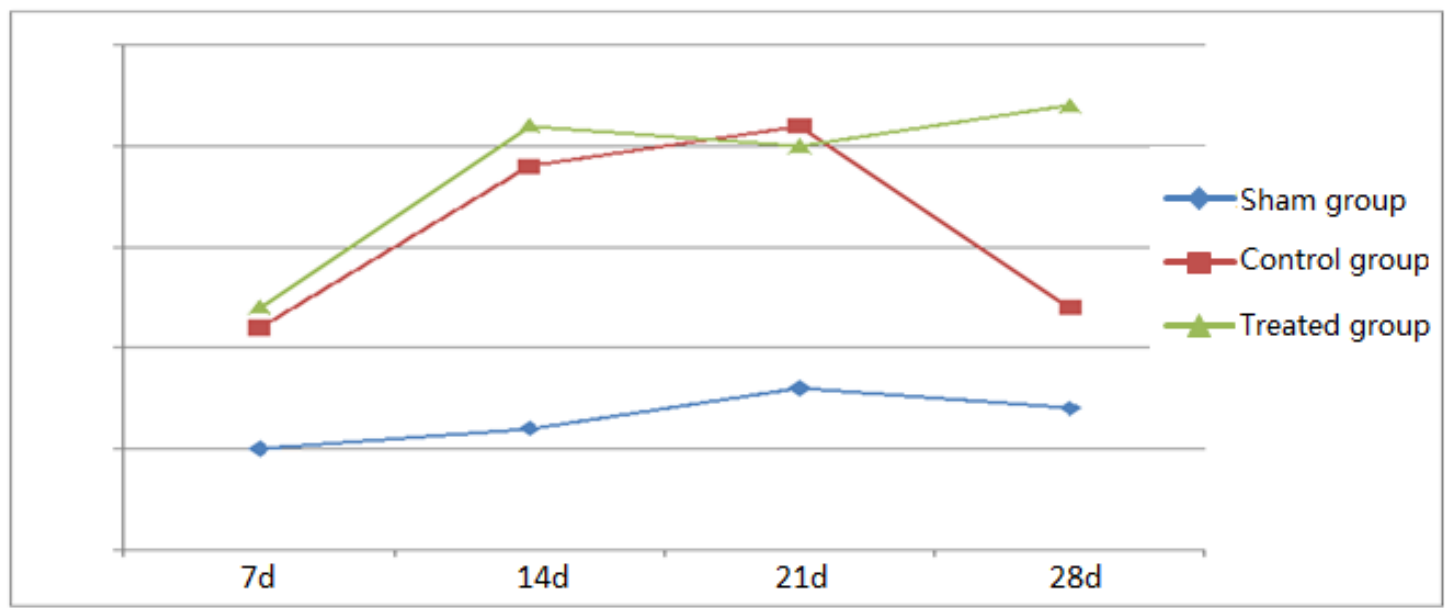

\subsection{Double Staining of GHR And Osteoclasts in Callus Tissue}

Immunohistochemical double staining showed that the region of GHR expression was highly consistent with that of osteoblasts 7, 14, 21 and 28 days after operation. However, there was no correlation between osteoclast staining areas (see Figure 4). In this experiment, the number and activity of osteoclasts in callus tissues of experimental rats at different stages were not detected.
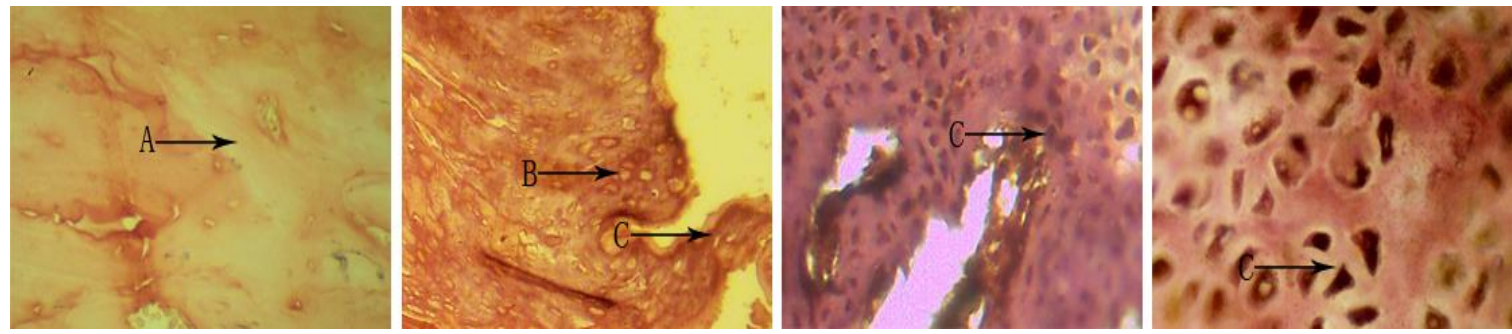

Figure 4. Double staining of GHR and osteoclasts

Bone matrix, B. osteoclasts, C. osteoblasts

\section{Discussion}

In the process of fracture healing, whether through intramembranous osteogenesis or interconal osteogenesis, bone tissue is formed by the proliferation and dense of mesenchymal stem cells (MSC), which are gradually differentiated into osteoblasts, which secrete osteoid and are embedded in them, then osteoid calcifies into osteoblast matrix and forms bone tissue [7]. Some bone growth factors can promote new bone formation and shorten fracture healing time [8]. Growth hormone $(\mathrm{GH})$ is the main hormone regulating substance metabolism. The main effect of $\mathrm{GH}$ on bone formation or reconstruction is to stimulate the formation of insulin-like growth factors (IGF-I and IGF-II). GH promotes the mitosis of bone cells, enhances the activity of ALP, promotes the synthesis of BGP, enhances the gene expression of osteonectin and promotes fracture healing. It promotes the differentiation and growth of growth plate chondrocytes. Improve intestinal calcium absorption, excite renal hydroxylase activity, and promote bone salt formation [9]. Promote the synthesis of chondroitin sulfate and collagen, accelerate cartilage calcification, effectively increase cortical bone mass and improve the overall 
mechanical properties of bone [10].

\subsection{ZJXG Decoction and Plasma GH Level and Fracture Healing}

After fracture, pituitary eosinophilic GH cells were in a high functional state [11]. The expression of GH was increased, and the serum GH level increased significantly within 24 hours, which affected the differentiation of osteoblasts, enhanced the activity of ALP, promoted the synthesis of osteocalcin, promoted gluconeogenesis, inhibited the degradation of sugar, and then increased the blood sugar level to compensate for the acute damage caused by trauma [12]. GH plays an important regulatory role in the process of early fracture healing, which may be involved in the initiation mechanism of early fracture healing. It has been reported that [13] GH can directly or indirectly act on osteoblasts and stimulate the differentiation, proliferation and maturation of osteoblasts at various stages. At the same time, $\mathrm{GH}$ is closely related to the formation of collagen I [14-16]. GH can also inhibit the formation and maturation of osteoclasts through IGF-I, induce osteoclasts to accelerate apoptosis [17], thereby promoting fracture healing. This suggests that early detection of GH level can effectively evaluate the prognosis of fracture. It also suggests that we should pay attention to the changes of GH level in patients with nonunion or delayed union of fracture, so as to find out the causes of nonunion or delayed union of fracture and take new treatment measures [18].

Animal experiments have shown that [19], exogenous growth hormone can promote fracture healing, and the healing time is one week ahead on average. However, the half-life of exogenous cytokines is short, the clearance rate is high, and the bioavailability of non-injection drugs is low. It cannot promote fracture healing effectively and continuously, which limits its clinical application [20]. Traditional Chinese medicine has a long history in the treatment of fracture, with remarkable curative effect and low price. Traditional Chinese medicine emphasizes the combination of drugs and systemic regulation. There is no complete theoretical support for whether traditional Chinese medicine can directly or indirectly affect the secretion, degradation and regulation of bone growth factor activity in the treatment of fracture, besides its conventional effects such as spasmolysis, pain relief, activating blood circulation and removing saprophytic muscles.

\subsection{ZJXG Decoction and GHR Expression Level of Callus and Fracture Healing}

GH can promote the growth of bone, cartilage and its tissues, stimulate the synthesis of protein and collagen, and promote the uptake and utilization of epoxy acid by tissue cells. However, the first step of GH's physiological function is to bind to GHR receptors on the surface of target cell membranes. GHR mediates the transmission of signals to human cells and produces a series of physiological effects [21]. Growth hormone receptor (GHR) is a transmembrane glycoprotein. It is a member of the haemopoietic cytokine receptor superfamily. There are about 620 amino acids. The exact number of amino acids varies slightly among different species. The molecular weight of GHR in most animals ranges from $100 \sim 130 \mathrm{kDa}$ [22]. GHR is abundant in liver and adipocytes, but GH receptors also exist in lymphocytes, fibroblasts, macrophages, chondrocytes, B2 insulin cells and osteoblasts [23].

Tartrate-resistant acid phosphatase is a good marker of bone resorption and osteoclast activity recently discovered. TRACP-5b is mainly derived from osteoclasts. The determination of serum TRACP- $5 b$ concentration is helpful to understand the physiological and pathological conditions of bone metabolism. Immunohistochemical staining showed that GHR of callus was mainly distributed in osteoblasts, so it can be inferred that GH could promote fracture healing mainly by enhancing the activity of osteoblasts. Whether GHR also exists in osteoclasts is not reported. 
Growth hormone receptors are expressed in many tissues of the body, but the expression levels are different, which may be an important reason for the asynchrony of different tissues, organs and overall development [24]. There is no relevant report about the effect of traditional Chinese medicine on GHR. Liu Li [25] et al. used reverse transcription polymerase chain reaction (RT-PCR) to quantitatively analyze the relative abundance of growth hormone receptor (GHR) mRNA in thymus and spleen of piglets. The results showed that the content of GHR mRNA in thymus and spleen of piglets was increased in different degrees by Si Jun Zi decoction. Immunohistochemical results showed that ZJXG Decoction did not significantly promote the expression of GHR receptor in callus in the early stage of fracture (1-3 weeks), but in the later stage (28 days), the expression of GHR was significantly higher than that in the control group. Whether it enhanced the expression of GHR or only enhanced its biological activity remains to be confirmed by further experiments.

\section{Conclusion}

ZJXG Decoction may promote fracture healing by enhancing GH activity, slowing down its degradation rate and enhancing GHR expression level in callus tissue at the later stage of fracture healing.

\section{Conflicts of Interest}

The authors declare that they have no Conflicts of Interest.

\section{Funding}

This experiment was supported by grants-in-aid for the Natural Science Fund of China $(81274116,81041092)$.

\section{Acknowledgements}

We thanks YuJinBio Sci. Tech. Co. Ltd. (www.yujinbio.com, Email: yujin@yujinbio.com) to help us edit our manuscript.

\section{Reference}

1. Raschke $\mathrm{M}$, Rasmussen $\mathrm{MH}$, Govender $\mathrm{S}$, et a1. Effects of growth hormone in patients with tibial fracture a randomized, double-blind, placebo-controlled clinical trial. Eur J Endocrinol, 2007, 156(3):341-351.

2. Tatsuyama $\mathrm{K}, \mathrm{Maezawa} \mathrm{Y}, \mathrm{Baba} \mathrm{H}$, et al. Expression of various growth factors for cell proliferation and cytodifferentiation during fracture repair of bone. European Journal of Histochemistry, 2009, 44(3):269278.

3. Gallea S, Lallemand F,Atfi A,et al. Activation of mitogen-acti-vated protein kinase cascades is involved in regulation of bone morphogenetic protein-2-induced osteoblast differentiation in pluripotent $\mathrm{C} 2 \mathrm{C} 12$ cells.Bone,2001, 28(5) :491-498.

4. Xun Sun, Shangqun Zhu,Structure, Function and Signaling Pathway of Growth Hormone Receptor Foreign Medical Sciences Section of Pathophysiology and Clinical Medicine,9-14

5. Huang Peijun. ZJXG Decoction combined with locking compression plate internal fixation for tibial plateau fractures. Inner Mongolia Traditional Chinese Medicine,2012,31(14): 20-21. 
6. Wang Xiangjie, Pan Yuexing, Du Zhixian, et al. Effects of ZJXG Decoction on the expression of bone morphogenetic protein-7 and neuropeptide $Y$ in rat fracture callus. Chinese Journal of Traditional Chinese Medicine, 2013, 28 (8): 2420-2422.

7. Zhao Huaizhi, Hao Hua, Lu Mingshu, et al. Quantitative study and application of callus on X-ray film. Chinese Journal of Orthopedics and Traumatology, 1995, 3(4): 8-9.

8. Turhani $D$, Weissenbock $M$, Stein $E$, et al. Exogenous recombinant human BMP-2 has little initial effects on human osteoblastic cells cultured on collagen type I coated/non coated hydroxyapatite ceramic granules. J Maxillofac Surg, 2007, 65(3):485-493.

9. Liu Haifeng, Chen Jinjie, Yang Peishun, et al. Study of Sanhua Jiegu Powder on Promoting Growth Hormone and Fracture Healing. Chinese Journal of Orthopedics and Traumatology, 1998, 6(2): 7-11.

10. Huang Suizhu, Dai Kerong, Tang Tingting, et al. Effects of recombinant human growth hormone on cortical bone in ovariectomized rats. Chinese Journal of Surgery, 2000,38(1): 18-22.

11. Gallea S,Lallemand F, Atfi A, et al. Activation of mitogen-acti-vated protein kinase cascades is involved in regulation of bone morphogenetic protein-2-induced osteoblast differentiation inpluripotent $\mathrm{C} 2 \mathrm{C} 12$ cells. Bone,2001, 28(5): 491-498.

12. Hedstrom M, Saf M, Brosjo $E$, et al. Positive effects of short-term growth hormone treatment on lean body mass and BMC after a hip fracture: a double-blind placebo-controlled pilot study in 20 patients. Acta Orthop scand, 2004, 75(4):394-401.

13. Takahashi M, Takahashi Y, Lida K, et al. Growth hormone stimulates tyrosine phosphorylation of local adhesion kinase (p125 (FAK)) and action stress fiber formation in human osteoblast-like cells. Saos2. Biochem Biopsy's Res Commun, 1999,263(1):100-106.

14. Andreasen TT, Oxlund H. Local anabolic effects of growth hormone on intact bone and healing fractures in rats. Calcif Tissuelnt, 2003, 73(3) :258-264.

15. Raschke $\mathrm{M}$, Rasmussen $\mathrm{MH}$, GovenderS, et al. Effects of growth hormone in patients with tibial fracture : a randomized, double-blind, placebo-controlled clinical trial. Eur J Endocrinol, 2007, 156(3) :341-351.

16. Takahashi MO, Takahashi Y, liila K, et al. Growth hormone stimulates tyrosine phosphorylation of focal adhesion kinase (P125FAK) and action stress fiber formation in human osteoblast-like cells, Saos2. Biochem Biophys Res Commun, 1999, 263(1):100-106.

17. Vestergaard P. Mosekilde. Fracture risk is decreased in acromegaly-a potential beneficial effect of growth hormone. Osteoporos Int, 2004,15(2):155-159.

18. Zhang Xiaolin, Sun Xiaolei, Ma Xinlong. Changes and clinical significance of serum growth hormone 7 insulin-like growth factor-I axis after long bone fracture. Chinese Journal of Trauma, 2012,28(5): 407411.

19. Chen Baoding, Li Qihua, Li Lihong, et al. Expressions of growth hormone and growth hormone receptor 
genes. Chinese Animal Husbandry and Veterinary Medicine, 2007, 34 (11): 52-54.

20. Gao Xue, Xu Shangzhong, Zhang Yinghan, et al. Research progress on the mechanism of growth hormone. Buffalo Magazine, 2003, 29 (1): 50-53.

21. Wang Yimin, Pei Guoxian, Wang Gang. Therapeutic effect of recombinant human growth hormone on radial small segment defect in rabbits. American Chinese Journal of Trauma, 2001, 4 (3): 36-38.

22. Wang Zengrong, Zhang Yonghong, Li Erfeng, et al. Nano-sustained-release system loaded with basic fibroblast growth factor and proliferation of bone marrow mesenchymal stem cells. China Tissue Engineering Research. 2012, 16 (47): 8794-8797.

23. Wang Junyue, Cheng fuzhen, Du Yuanbin, et al. Effect of Jiegu Qili tablet on experimental fracture healing. Chinese Journal of Orthopedics and Traumatology, 1997, 5 (2): 45-47.

24. Xia Dong, Zhao Ruqian. Developmental changes and growth characteristics of growth hormone receptor gene expression in pig stomach tissue. Chinese Journal of genetics, 2002,29 (6): 492-496.

25. Liu Li, Hao Fuxing, Mao haiYin. Sijunzi Decoction on Growth Hormone Receptor (GHR) mRNA Expression in Thymus and Spleen of Piglets. Advances in Animal Medicine, 2009, 30(6):66-69. 